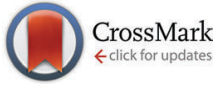

Cite this: DOI: $10.1039 /$ c6cc08621g

Received 26th October 2016

Accepted 29th November 2016

DOI: $10.1039 / \mathrm{c6cc08621g}$

www.rsc.org/chemcomm

\title{
Single molecule based SNP detection using designed DNA carriers and solid-state nanopores $\dagger$
}

\author{
Jinglin Kong, Jinbo Zhu and Ulrich F. Keyser*
}

\begin{abstract}
Single nucleotide polymorphisms (SNPs) play a crucial role as molecular markers in medical and diagnostic application. We demonstrate a nanopore based method for SNP detection at the single molecule level. Designed DNA carriers are used to distinguish DNA strands containing only one single base difference and follow strand displacement kinetics.
\end{abstract}

With the developing knowledge of the human genome, DNA variants have been pinpointed to a large number of diseases. The most common type of the variations is single nucleotide polymorphisms (SNPs). ${ }^{1}$ Methods that are able to discriminate a single mismatch in a DNA strand are thus of crucial importance in medical research, disease diagnosis and drug discovery. Major traditional detection methods include mass spectrometry, fluorescence and chemiluminescence. ${ }^{2}$ Although considerable improvements have been made towards high detection sensitivity ${ }^{3}$ and throughput, ${ }^{4}$ novel methods, especially those aiming at low detection concentration, small sample volume and single molecule readout, are in great demand.

Solid-state nanopores have emerged as a versatile single molecule technique in biosensing. ${ }^{5,6}$ Considering the advantages including low detection limit, rapid analysis and potential of simultaneous screening, nanopore-based methods are attractive candidates for nucleic acid analysis. ${ }^{7-9}$ Sequence specific recognition has been demonstrated by using PNA, ${ }^{10}$ protein $^{11}$ probes or immobilizing DNA strands onto nanopores. ${ }^{12-14}$ Short oligonucleotides labeled with proteins ${ }^{15}$ or nanoparticles ${ }^{16}$ were used to discriminate between hybridized and single-stranded nucleic acids. However, only few studies have shown SNP discrimination at the single molecule level. ${ }^{14,16}$

In this work, we combine smart designed DNA carriers and glass nanopores for highly specific and adaptable SNP detection. DNA carriers have been introduced for specific protein detection previously. ${ }^{17-19}$ In this work, the DNA carrier platform was adapted for SNP detection (Fig. 1a). A disease related Janus

Cavendish Laboratory, University of Cambridge, Cambridge, UK.

E-mail: ufk20@cam.ac.uk

$\dagger$ Electronic supplementary information (ESI) available. See DOI: 10.1039/c6cc08621g kinase 2 (JAK2) V617F mutation was chosen as a model system. ${ }^{20}$ JAK2 is widely referenced as a biomarker of myeloproliferative disorders (MPDS). ${ }^{21,22}$ The point mutation from $\mathrm{G}$ to $\mathrm{C}$ disrupts the auto-inhibitory activity of the JAK2 protein and therefore produces abnormal blood cells. ${ }^{23}$ As shown in Fig. 1b the complementary sequence for the point mutation JAK2 gene is integrated into the centre of the DNA carrier as an overhang (strand S95F). The last 6 bases (blue) with the mutation base
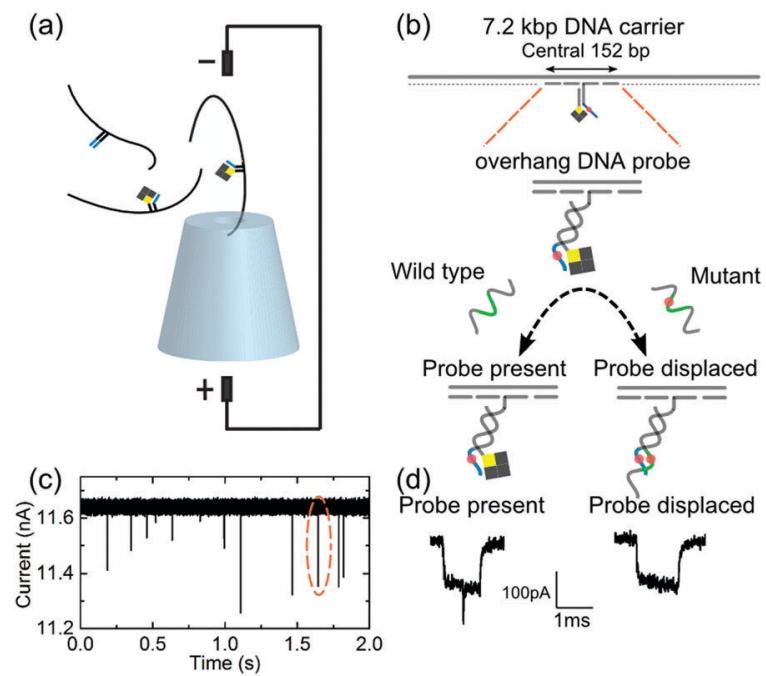

Fig. 1 SNP detection using DNA carriers and nanopore sensing. (a) Schematic of DNA carriers (blank lines) translocating through a nanopore driven by the electric field. (b) Schematic of the designed DNA carrier for SNP detection. A pair of overhang DNA probes is located in the centre of the carrier. The central 152 bp section is enlarged to show the displacement initialized by target DNA strands. The 6 nucleotides toehold design is marked in blue in the DNA probes and in green in the target strands. The SNP on the mutant strand is shown as a pink spot and the signal-producing streptavidin is shown as squares with the yellow part representing the only active binding site. (c) Example ionic current trace recorded during nanopore measurements. An event appears as a current drop when a DNA carrier translocates through the nanopore. (d) Zoomed events from (c). The two examples show the typical events when the signal probe is present or displaced. 
marked as a pink point at the $5^{\prime}$ end are designed as a toehold for initiating the displacement reaction. The signal producing probe containing a $3^{\prime}$ biotin modification is initially hybridized to the DNA carrier. The attached monovalent streptavidin ${ }^{24}$ (yellow and grey squares) produces a secondary current drop in the ionic current signal (Fig. 1c and d). After incubation, only the perfectly matching JAK2 mutant strand is able to displace the signal probe. This reaction will remove the streptavidin from the DNA carrier and thus take out the secondary current drop within the translocation event. Example translocation events with the probe present or displaced are shown in Fig. 1d. The fraction of the occupied events allows for the estimation of the proportion of the strand-displacement that has been completed.

Firstly, the capability of designed DNA probes for SNP detection is validated by a traditional fluorescence-based assay. The overhang part of the on carrier probe is adapted to a fluorescent probe, by modifying the $3^{\prime}$ end with a FAM label (Fig. 2a). As shown in the fluorescent spectrum (Fig. 2b), the fluorescent signal is initially quenched by the quencher probe, strand $\mathrm{Q}(0 \%$ control, grey). With the addition of the mutant or wild type strands, fluorescence is recovered as some of the quencher probes are displaced (red and blue). The $100 \%$ control (black) is obtained by hybridizing the fluorescent probe with a mutant strand. Clear differences are observed between the mutant and wild type strands which prove that the single mismatched base in the toehold domain strongly affected the efficiency of the strand displacement reaction.

We used gel electrophoresis as an alternative evaluation assay. The same set of DNA probes and target strands as in the fluorescent assay were used. The gel image (Fig. 2c) shows an almost complete displacement (lane 5) by the mutant strands while most of the FQ duplex remained after the addition of the wild type strands (lane 4). The well-defined bands in lane 3 and lane 6 are markers showing

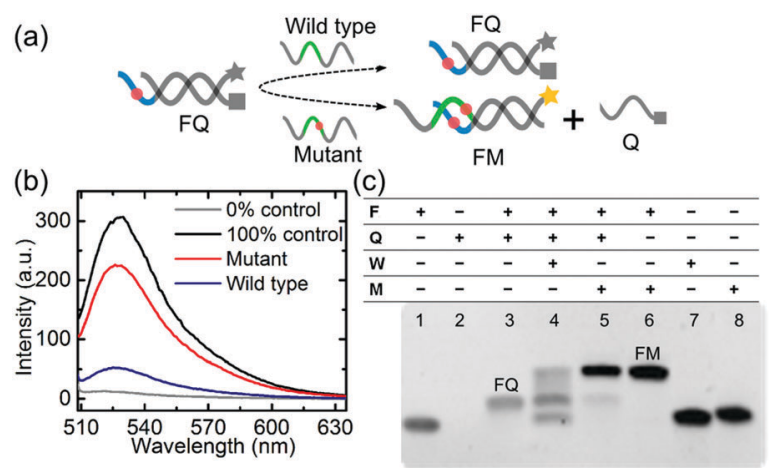

Fig. 2 Validation of strand displacement reaction with bulk assays. (a) Schematic of the DNA displacement principle upon the presence of mutant or wild type target strand. The fluorescent label is shown as a star and the quencher is shown as a grey square. DNA strand sequences are given in ESI, $\dagger$ Table S1. (b) Fluorescent spectra of DNA oligo probes incubated with mutant or wild type target strands. The '0\%' control shows the fluorescent signal from only the hybridized probe pair, namely $F$ and $Q$, and the ' $100 \%$ ' control is obtained by having the fluorescent labeled probe, hybridized with the fully matched mutant strand. Repeats are shown in $\mathrm{ESI}, \dagger$ S5. (c) Results of the gel electrophoresis characterization. 15\% PAGE was run at $110 \mathrm{~V}$ with $1 \times$ TBE buffer. The sample information of the 8 lanes is shown in the table above accordingly. the initially hybridized FQ remains in lane 4 and FM formed after the strand displacement in lane 5, respectively. The agreement of these assays indicates the capability of the designed probes for SNP discrimination.

Based on the bulk validation, we hybridized the designed probes (strand S95F) onto the DNA carrier, positioned in the middle of the single stranded scaffold. The signal probe is hybridized with the DNA carrier during synthesis, making up the full unit part of a JAK2 carrier. The $3^{\prime}$ biotin label on probe $\mathrm{Q}$ is there to bind a relatively large streptavidin $(\sim 53 \mathrm{kDa})$ and thus enhance the current drop signal. Compared to the previous signal producing strategies, such as the DNA dumbbells, ${ }^{19}$ only a single site modification is needed to create the current signature, which offers more flexibility and simplicity for complex DNA carrier based detection protocols in the future.

First of all, we demonstrate the ability of the DNA carrier technique to follow DNA displacement kinetics measurements. The JAK2 carriers were mixed with mutant or wild type strands, and immediately transferred into the fluidic chip ${ }^{25}$ for translocation measurements. The ' $0 \%$ ' control is obtained by having only the JAK2 carrier in the sample. The ionic current recording was kept at $600 \mathrm{mV}$ bias voltage and run continuously for $\sim 2$ hours. The displacement kinetics are analyzed by splitting all linear translocation data into 50 events subgroups in the time order. The time evolvement of occupied fractions is plotted in Fig. 3a. In case the nanopore was clogged during the 2 hours of recording, the electrodes were immediately moved to another nanopore. The time period while changing is shown as discrete points in the plot. Ten times concentrated target strand was added in the kinetics study to assure clear changes can be observed in the 2 hours recording window.

A clear decrease in occupied fraction is found with the mutant sample, indicating the increasing proportion of the signal probes being displaced. The occupied fraction changes from $\sim 0.90$ to $\sim 0.25$, which is close to the full dynamic range of a previously studied similar system. ${ }^{18}$ Event subgroups at different time points in the measurements are shown in Fig. 3 b. In the beginning, 8 out of 10 events show the occupied ionic current signature while after 60 minutes and 130 minutes, the numbers of occupied event decrease to 6 and 3 respectively, indicating the DNA displacement reaction happens continuously. As for the wild type sample, the occupied fraction is almost kept the same, with no clear difference from the ' $0 \%$ ' control. In order to benchmark our results, we performed real time fluorescent assays for comparison. As shown in the inset of Fig. 3a, the recovered fluorescence increases over time. The contrast between the mutant and wild type sample is obvious.

The ability of measuring the strand displacement is highly desirable, since it provides more insights into the reaction which could further facilitate observations of other nanoscale interactions with active behaviours, such as catalysts and nanomotors. ${ }^{8,26,27}$ Additionally, the single molecule readout offers high accuracy and low detection limit compared to the traditional bulk measurements. Compared to fluorescent-based assays, the working concentration of DNA carriers is lowered down to $2 \mathrm{nM}$ while the sample volume in the microfluidic chip is only $10 \mu \mathrm{L}$, thus the 
(a)

(b)

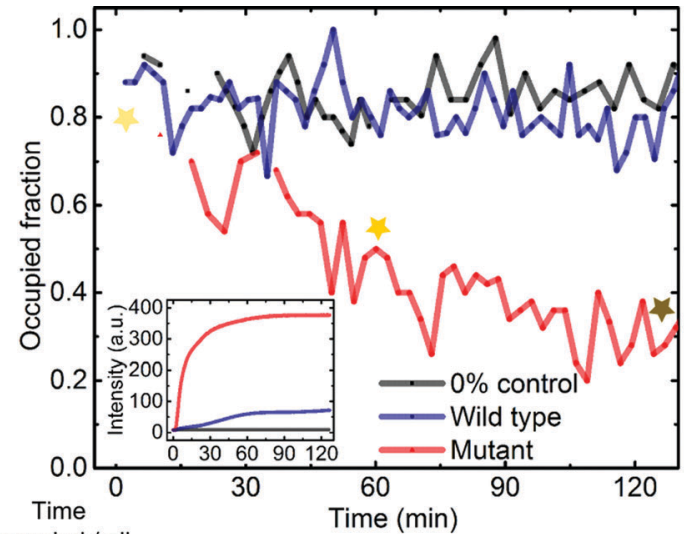

occupied / all

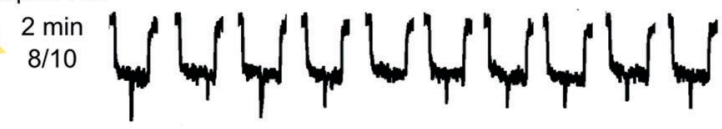

$60 \mathrm{~min}$ $6 / 10$
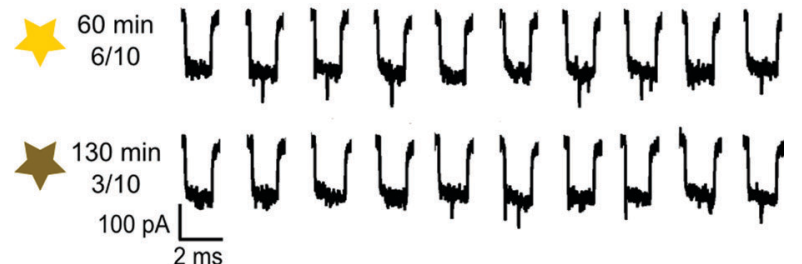

Fig. 3 Single molecule kinetics. (a) DNA displacement kinetics inferred from nanopore measurements. The occupied fraction of each data point is obtained from a group of 50 events over time. The concentration of DNA carrier was $2 \mathrm{nM}$ while the target strand was chosen to be $20 \mathrm{nM}$. $4 \mathrm{nM}$ streptavidin was added $\sim 5$ minutes before the measurements. The total event number in the plot is 2220, 2910 and 2130 for control, wild type and mutant sample, respectively. The inset shows DNA displacement kinetics using fluorescence-based measurements. All measurements were performed in aqueous solution containing $4 \mathrm{M} \mathrm{LiCl}, 100 \mathrm{mM} \mathrm{NaCl}, 10 \mathrm{mM} \mathrm{KCl}$, $10 \mathrm{mM} \mathrm{MgCl}_{2}$ buffered with TE (pH 7.5). (b) Example 10 -event subsets at different time points during the 2 hours kinetics measurements. In the beginning (after 2 minutes), 8 out of 10 events show a secondary current drop, while after 60 and 130 minutes, the number of positive events drops to 6 and 3 out of 10 respectively. The stars in corresponding colors are marked in (a) indicating the time points where the subgroups come from.

total amount of DNA needed is only $\sim 20$ femtomole. Fig. 3a shows data analyzed from $\sim 2500$ linear translocation events in each plot, which means the sample concentration can be even lower with further practical optimization such as multi-channel recording in parallel $^{25}$ and microfluidic designs with smaller volume.

Having investigated the capability of SNP discrimination using the nanopore-DNA carrier method, we tested the target concentration dependence of the occupied fraction. Mutant and wild type strands at the concentration of $2 \mathrm{nM}, 6 \mathrm{nM}, 10 \mathrm{nM}$, $15 \mathrm{nM}$ and $20 \mathrm{nM}$ were incubated with $2 \mathrm{nM}$ DNA carriers for 2 hours before the measurements. After the incubation, the occupied fraction becomes stable and does not change anymore during the measurements (ESI, $\dagger$ S7). As shown in Fig. 4a, the averaged occupied fraction decreases along the increase of mutant strands concentration: from $0.63 \pm 0.067$ at 1 time mutant strands to $0.25 \pm 0.050$ at 10 times mutant strands. The linear trend indicates the potential as a quantitative application of the method. However, the trend is not that clear with the wild
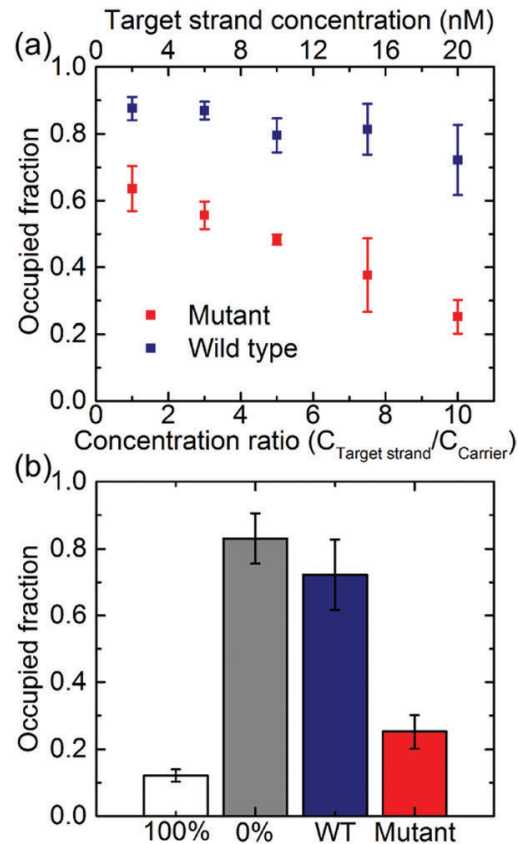

Fig. 4 Distinguish single-mismatch using DNA carrier and nanopores. (a) Occupied fraction dependence over a range of target strand concentrations. The plot is obtained from a total number of 41 nanopores. The event numbers for data point at concentration ratio of 1, 3, 5, 7.5 and 10 are 921 , $856,886,564$ and 1711 for the wild type strand, and 2278, 1562, 1369, 1149, 1309 for mutant strand (detailed statistics in ESI, $\dagger$ S11). Errors are standard deviations. The incubation was carried out in $100 \mathrm{mM} \mathrm{NaCl}, 10 \mathrm{mM} \mathrm{KCl}$, $10 \mathrm{mM} \mathrm{MgCl} 2$ buffered with TE ( $\mathrm{pH} \sim 8)$, at room temperature for 2 hours. $4 \mathrm{nM}$ streptavidin was added $\sim 5$ minutes before the measurements. (b) Occupied fraction comparison of DNA carriers incubated with mutant strand and wild type (WT) strand. The '100\%' control is prepared by having the mutant strand added during DNA carrier synthesis. The ' $0 \%$ ' control is from a fully labeled DNA carrier before strand displacement. Occupied fractions of wild type and mutant samples shown here are incubated with 10 times target DNA strand over the carrier. Errors are standard deviations.

type sample: the occupied fraction fluctuates around the background level, ranging from $0.87 \pm 0.034$ to $0.72 \pm 0.11$. Errors are standard deviations.

The biggest difference in strand displacement is found with 10 times excess target strands, but a significantly detectable difference is already clear at 1 to 1 ratio of target and DNA carriers. The comparison of occupied fraction of the controls and DNA carrier incubated with 10 times mutant and wild type strands is shown in Fig. 4b. The ' $100 \%$ ' control (white) shows a $\sim 0.1$ false positive signal which most likely originates from the presence of DNA knots ${ }^{28}$ on bare DNA carriers, and the $0 \%$ control (grey) levels off at $\sim 0.85$ instead of 1 resulting from a small fraction of imperfect DNA carriers without the biotin label. ${ }^{18}$ However, a flexible sample concentration window can be targeted due to the single molecule nature of this nanoporebased method. Since the translocation behavior is dominated by the DNA carrier, the sample concentration being measured can be tuned by varying the carrier concentrations ( $2 \mathrm{nM}$ is used in this work). For target samples with high concentration, more carriers can be used for better statistics and faster detection. For lower target concentrations, less concentrated DNA carriers 
would still maintain the contrast between the mutant and wild type strands.

In this work, we have shown that our method of DNA carriers and nanopore sensing can be used for highly specific and sensitive SNP detection. With the JAK2 gene sequence as a model system, we show the capability of this method to detect SNPs at nanomolar concentration. The occupied fraction is related to the concentration of the target DNA strands, and can be used to follow the DNA displacement kinetics over time. Our method of using designed DNA carrier has several potential advantages over the existing approaches. First, the nature of the DNA carrier platform makes it convenient to be adapted for other nucleic acids detection including DNA biomarkers, mRNA and rRNA. Previous methods always involve surface immobilization or conjugation steps. Modification chemistry can sometimes be challenging and not favourable for detection specificity especially at the single molecule level. In contrast, our design with DNA is much more precise due to the specific basepairing rules. Second, the giant DNA carrier, compared with the short oligo used previously, plays the role of a molecule 'ruler' to help selecting only the defined translocation event in data analysis, which dramatically increases the detection accuracy. Moreover, the 190 designable oligonucleotides offer opportunities for the structure to carry more information. Its potential for multiplexed ${ }^{19}$ nucleic acids sensing could bring new approaches to improve the detection speed and throughput.

The authors thank the Howarth Lab, Oxford for providing the monovalent streptavidin sample, N. A. W. Bell and K. Chen for useful discussions, E. A. Hemmig and M. Ricci for critical reading of the manuscript. J. Kong acknowledges funding from Chinese Scholarship Council and Cambridge Trust. J. Zhu and UFK acknowledge support from an ERC Consolidator grant (DesignerPores 647144).

\section{References}

1 R. Sachidanandam, D. Weissman, S. C. Schmidt, J. M. Kakol, L. D. Stein, G. Marth, S. Sherry, J. C. Mullikin, B. J. Mortimore and D. L. Willey, Nature, 2001, 409, 928-933.

2 S. Kim and A. Misra, Annu. Rev. Biomed. Eng., 2007, 9, 289-320.
3 S. X. Chen, D. Y. Zhang and G. Seelig, Nat. Chem., 2013, 5, 782-789.

4 C. P. Schaaf, J. Wiszniewska and A. L. Beaudet, Annu. Rev. Genomics Hum. Genet., 2011, 12, 25-51.

5 U. F. Keyser, Nat. Nanotechnol., 2016, 11, 106-108.

6 E. C. Yusko, J. M. Johnson, S. Majd, P. Prangkio, R. C. Rollings, J. Li, J. Yang and M. Mayer, Nat. Nanotechnol., 2011, 6, 253-260.

7 M. Wanunu, T. Dadosh, V. Ray, J. Jin, L. McReynolds and M. Drndić, Nat. Nanotechnol., 2010, 5, 807-814.

8 Y. L. Ying, H. Y. Wang, T. C. Sutherland and Y. T. Long, Small, 2011, 7, 87-94.

9 B. M. Venkatesan and R. Bashir, Nat. Nanotechnol., 2011, 6, 615-624.

10 A. Singer, M. Wanunu, W. Morrison, H. Kuhn, M. Frank-Kamenetskii and A. Meller, Nano Lett., 2010, 10, 738-742.

11 J.-S. Yu, M.-C. Lim, D. T. N. Huynh, H.-J. Kim, H.-M. Kim, Y.-R. Kim and K.-B. Kim, ACS Nano, 2015, 9, 5289-5298.

12 S. Howorka, S. Cheley and H. Bayley, Nat. Biotechnol., 2001, 19, 636-639.

13 S. Hernández-Ainsa, N. A. Bell, V. V. Thacker, K. Göpfrich, K. Misiunas, M. E. Fuentes-Perez, F. Moreno-Herrero and U. F. Keyser, ACS Nano, 2013, 7, 6024-6030.

14 S. M. Iqbal, D. Akin and R. Bashir, Nat. Nanotechnol., 2007, 2, 243-248.

15 O. K. Zahid, F. Wang, J. A. Ruzicka, E. W. Taylor and A. R. Hall, Nano Lett., 2016, 16, 2033-2039.

16 Y. S. Ang and L.-Y. L. Yung, ACS Nano, 2012, 6, 8815-8823.

17 N. A. Bell and U. F. Keyser, J. Am. Chem. Soc., 2015, 137, 2035-2041.

18 J. Kong, N. A. Bell and U. F. Keyser, Nano Lett., 2016, 16, 3557-3562.

19 N. A. Bell and U. F. Keyser, Nat. Nanotechnol., 2016, 11, 645-651.

20 J. Zhu, L. Zhang, S. Dong and E. Wang, Chem. Sci., 2015, 6, 4822-4827.

21 A. V. Jones, S. Kreil, K. Zoi, K. Waghorn, C. Curtis, L. Zhang, J. Score, R. Seear, A. J. Chase and F. H. Grand, Blood, 2005, 106, 2162-2168. 22 I. Warshawsky and F. Mularo, J. Clin. Pathol., 2011, 64, 905-910.

23 R. Kralovics, F. Passamonti, A. S. Buser, S.-S. Teo, R. Tiedt, J. R. Passweg, A. Tichelli, M. Cazzola and R. C. Skoda, N. Engl. J. Med., 2005, 352, 1779-1790.

24 M. Howarth, D. J. Chinnapen, K. Gerrow, P. C. Dorrestein, M. R. Grandy, N. L. Kelleher, A. El-Husseini and A. Y. Ting, Nat. Methods, 2006, 3, 267-273.

25 N. A. Bell, V. V. Thacker, S. Hernández-Ainsa, M. E. Fuentes-Perez, F. Moreno-Herrero, T. Liedl and U. F. Keyser, Lab Chip, 2013, 13, 1859-1862.

26 H.-Y. Wang, Z. Gu, C. Cao, J. Wang and Y.-T. Long, Anal. Chem., 2013, 85, 8254-8261.

27 D. Zhang and E. Winfree, J. Am. Chem. Soc., 2009, 131, 17303-17314.

28 C. Plesa, D. Verschueren, S. Pud, J. van der Torre, J. W. Ruitenberg, M. J. Witteveen, M. P. Jonsson, A. Y. Grosberg, Y. Rabin and C. Dekker, Nat. Nanotechnol., 2016, DOI: 10.1038/nnano.2016.153. 